\title{
A highly contiguous genome assembly of Brassica nigra (BB) and revised nomenclature for the pseudochromosomes
}

\author{
Kumar Paritosh $^{1}$, Akshay Kumar Pradhan ${ }^{1,2}$, Deepak Pental ${ }^{1^{*}}$ \\ ${ }^{1}$ Centre for Genetic Manipulation of Crop Plants, University of Delhi South Campus, New Delhi 110021, India \\ ${ }^{2}$ Department of Genetics, University of Delhi South Campus, New Delhi110021, India
}

*To whom correspondence should be addressed

Tel. +91-11-24116392

Fax. +91-11-24116392

Email: dpental@gmail.com

Running title: Chromosome-scale genome assembly of Brassica nigra 


\begin{abstract}
Brassica nigra (BB), also called black mustard, is grown as a condiment crop in India. $B$. nigra represents the $\mathrm{B}$ genome of U's triangle and is one of the progenitor species of $B$. juncea $(\mathrm{AABB})$, an important oilseed crop of the Indian subcontinent. We report here a highly contiguous genome assembly of B. nigra variety Sangam. The genome assembly has been carried out using Oxford Nanopore long-read sequencing and optical mapping. The resulting chromosome-scale assembly is a significant improvement over the previous draft assemblies of $B$. nigra; five out of the eight pseudochromosomes were represented by one scaffold each. The assembled genome was annotated for the transposons, centromeric repeats, and genes. The B. nigra genome was compared with the recently available contiguous genome assemblies of B. rapa (AA), B. oleracea (CC), and B. juncea (AABB). Based on the maximum homology among the three diploid genomes of U's triangle, we propose a new nomenclature for $B$. nigra pseudochromosomes, taking the $B$. rapa pseudochromosome nomenclature as the reference.
\end{abstract}

Keywords - Brassica nigra, genome assembly, gene blocks, pseudochromosome nomenclature, evolution 


\section{Introduction}

Nagaharu ${ }^{1}$ based on his observations and preceding cytogenetic work ${ }^{2}$ proposed a model on the relationship of some of the cultivated Brassica species. The model, known as U's triangle, described the relationship of three diploid species - B. rapa (Bra, AA, n=10), B. nigra $(\mathrm{Bni}, \mathrm{BB}, \mathrm{n}=8)$ and $B$. oleracea $(\mathrm{Bol}, \mathrm{CC}, \mathrm{n}=9)$ with three allopolyploid species $-B$. juncea (Bju, AABB, n=18), B. napus (Bna, AACC, $\mathrm{n}=19$ ) and B. carinata (Bca, BBCC, $\mathrm{n}=17$ ). Subsequent cytogenetic work on inter-specific and inter-generic hybrids between the Brassica species of the U's triangle and other taxa in the tribe Brassiceae showed close relationships and the group was described as Brassica coenospecies ${ }^{3,4}$.

Since the early cytogenetic work, major insights have been gained into the evolution of the Brassica species based on the extent of nucleotide substitutions in the orthologous genes belonging to the nuclear ${ }^{5}$ and plastid genomes ${ }^{6,7,8,9}$, analysis of genome synteny using molecular markers ${ }^{10,11}$, in situ hybridizations ${ }^{12}$, and genome sequencing ${ }^{13,14,15,16}$. The most significant observation is that the three diploid species of the U's triangle - B. rapa, B. nigra, B. oleracea, and the other diploid species belonging to the tribe Brassiceae have originated through genome triplication, referred to as the $\boldsymbol{b}$ event ${ }^{5}$. Genome triplication was followed by extensive chromosomal rearrangements leading to gene block reshuffling vis-à-vis the gene block order in Arabidopsis thaliana (At) ${ }^{17,18}$, and gene fractionation due to a differential loss of genes in the three constituent paleogenomes ${ }^{19}$. The diploid species of the tribe Brassiceae are, therefore, mesohexaploids. It is now accepted that tribe Brassiceae is defined by the $\boldsymbol{b}$ event; it is, however, not clear whether the $\boldsymbol{b}$ event happened once or more times. The presence of two plastid lineages ${ }^{6,7,8,9}$ points to a minimum of two independent $\boldsymbol{b}$ events ${ }^{20}$.

Genome assemblies of B.rapa ${ }^{13}$, B. $_{\text {oleracea }^{14}}$, B. napus $^{15}$, and B. juncea ${ }^{16}$ were first reported using short-read Illumina sequencing. More recent assemblies of these species have used long-read sequencing technologies, either PacBio SMRT (single-molecule real-time) sequencing or Oxford Nanopore Technologies (ONT) ${ }^{21,22,23}$. Scaffolding has been carried out with optical mapping and/or Hi-C technologies. The most extensive assembly of the B genome has been made available from our recent effort on the genome assembly of an oleiferous type of $B$. juncea variety Varuna with SMRT sequencing and optical mapping ${ }^{23}$.

We report here a highly contiguous genome assembly of $B$. nigra variety Sangam, a photoperiod insensitive, short-duration variety, grown under dryland conditions, and used as 
a seed condiment crop in India. The assembly has been carried out using Nanopore sequencing and optical mapping. Previously reported Illumina short-read sequences and a genetic map of $B$. nigra $^{23}$ were used for error correction and assigning the contigs and scaffolds to the eight pseudochromosomes. We compared the structure of the B genome of $B$. nigra (BniB) with the genomes of B. rapa $^{21}$ (BraA), B. oleracea ${ }^{22}$ (BolC), and also the B genome of $B$. juncea $^{23}$. (BjuB). We propose a revised nomenclature for the B. nigra pseudochromosomes based on maximum homology between the $\mathrm{A}$ and $\mathrm{B}$ genome pseudochromosomes; the $B$. rapa A genome nomenclature being the reference as it was the first Brassica genome that was sequenced ${ }^{13}$.

\section{Materials and methods}

\subsection{Plant material, genome size estimation, nanopore sequencing, optical mapping, and genome assembly}

A DH (doubled haploid) line BnSDH-1 of Brassica nigra variety Sangam ${ }^{23}$ was used for genome sequencing and assembly. BnSDH-1 was maintained by bud pollination. For DNA isolation, BnSDH-1 seedlings were grown in a growth chamber maintained at $8 \mathrm{~h}$ light, $25^{\circ} \mathrm{C} / 16 \mathrm{~h}$ dark, $10^{\circ} \mathrm{C}$ cycle. DNA was isolated from the leaves of $10 \mathrm{~d}$ old seedlings; the harvested leaves were immediately frozen in liquid nitrogen. High molecular weight DNA was isolated from the leaf tissues by CTAB method ${ }^{24}$. For Nanopore sequencing, genomic DNA libraries were prepared using the 'Ligation sequencing kit 1D' following the manufacturer's instructions (Oxford Nanopore). In brief, around $2 \mu \mathrm{g}$ of high molecular weight DNA was repaired using the 'NEBNext FFPE DNA Repair mix' and the 'Ultra 11 End-prep Enzyme mix'; subsequently, the adapter mix was ligated to the repaired DNA using the 'NEBNext Quick T4 DNA Ligase'. At the end of each step, DNA was cleaned with the 'AMPure XP beads' (Thermo Fisher Scientific). The quality and quantity of the DNA libraries were determined with a Nanodrop spectrophotometer. DNA libraries were sequenced on the MinION device using the MinION Flow Cells R 9.4.1 (Oxford Nanopore). Base-calling and quality filtering were carried out using Albacore software (https://github.com/Albacore). Previously generated ${ }^{23}$ Illumina short-read sequencing data ( 100x coverage) of the line BnSDH-1 was used at various steps (described wherever used) of the new genome assembly. Approximately 40x Illumina PE (2x100 bp) data with a kmer length of 21 was used for the kmer frequency distribution analysis with Jellyfish v2.2.6 $6^{25}$. 
The output histogram file was used to estimate the genome size of BnSDH-1 using the findGSE $\operatorname{program}^{26}$.

Raw Nanopore reads were assembled into contigs using the Canu assembler V1.6 ${ }^{27}$ with the parameters 'minRead length' and 'minOverlap length' set at values of $1000 \mathrm{bp}$. The pairedend $(\mathrm{PE})$ reads obtained earlier with Illumina sequencing $(\sim 100 \mathrm{x}$ coverage) were mapped on the assembled Nanopore contigs using BWA-MEM ${ }^{28}$, followed by error correction with the Pilon program ${ }^{29}$ in five iterative cycles. After each of the Pilon cycle, completeness of the corrected genome was ascertained with Benchmarking Universal Single Copy Orthologue (BUSCO) program $(\mathrm{V} 4.0 .5)^{30}$. OrthoDB v10 plant datasets were used as the reference for analyzing the completeness of the predicted genes.

Optical mapping was carried out following the protocols suggested by the manufacturer (Bionano Genomics). Leaf tissues from $7 \mathrm{~d}$ old seedlings were harvested and transferred to an ice-cold fixing solution. Nuclei were isolated using the 'rotor-stator' protocol (Bionano genomics, Document no: 30228) and the nuclear fraction was purified on a sucrose density gradient. The nuclei were embedded in $0.5 \% \mathrm{w} / \mathrm{v}$ agarose followed by treatment with proteinase-K (Qiagen) for $2 \mathrm{~h}$. Mapping was carried out with three different labeling reactions - two NLRS (Nicks, Labels, Repairs and Stains) and one DLS (Direct Label and Stain). For the two NLRS labeling reactions, agarose plugs were treated with BssSI and BspQI and the nicks were labeled with the 'IrysPrep NLRS labeling kit'. In the DLS labeling reaction, DNA was recovered from the agarose plugs, suspended in TE buffer, and labeled with the 'Bionano Prep DLS kit'. Mapping data were obtained from the labeled libraries on the Saphyr system (Bionano) using one lane for each library. Mapping and hybrid assemblies were performed using the Bionano Access software. A previously generated genetic map of B. nigra ${ }^{23}$, developed using an $\mathrm{F}_{1} \mathrm{DH}$ population from a cross of line BnSDH- $1 \times$ line 2782 was used for validating the scaffold level assemblies and assigning the scaffolds to the eight linkage groups (LGs) to constitute eight pseudochromosomes. Position of the GBS marker tags was determined on the scaffolds with a Blastn search analysis. A correlation plot of the physical and genetic position of the markers was developd to validate the integrity and quality of scaffolding. Scaffolds were positioned and oriented on each pseudochromosome based on the information obtained with the correlation plots.

\subsection{Transcriptome sequencing, gene, and transposon annotation}


Illumina short-read based transcriptome sequencing of the line BnSDH-1 has been reported earlier ${ }^{23}$. The same protocol was used for transcriptome sequencing of the line 2782, an East European gene pool line of B. nigra. For transcriptome sequencing of the line BnSDH-1 on the PacBio platform, total RNA was isolated from the seedling, leaf, and developing inflorescence tissues using the 'Spectrum plant total RNA kit' (Sigma). The quality of the RNA was checked with Bioanalyzer 2100 using the 'RNA 6000 Nano kit' (Agilent). RNA samples with RIN values $>7$ were used for further analysis. Transcriptome sequencing was carried out on the pooled RNA. Three different libraries of the size range $0.5-1 \mathrm{~kb}, 1-2 \mathrm{~kb}$, and $2-6 \mathrm{~kb}$ were prepared using the 'SMRTbell Template Prep kit' and sequenced on a PacBio RS II sequencer. The raw sequences obtained from each of the three libraries were assembled separately using SMRT Analysis software (v1.4). Full-length nonchimeric sequences were used for clustering with ICE (Structure Clustering and Error Correction) algorithm; partial reads were used for polishing of the ICE generated consensus sequences. ORFs were predicted from the polished consensus sequences using the ANGEL software (https://github.com/PacificBiosciences/ANGEL).

Transposable elements (TEs) were identified in the genome assembly using the Repeatmodeler pipeline (http://www.repeatmasker.org/RepeatModeler/). A de-novo repeat library was developd using RECON, RepeatScout and Tandem Repeat Finder programs available in the Repeatmodeler pipeline, and NSEG (ftp://ftp.ncbi.nih.gov/pub/seg/nseg/) program. The developed TE library, along with the repbase database for At was used to predict TEs in the assembled genome using RepeatMasker (htntp://www.repeatmasker.org). Identified LTR sequences were validated by the LTR finder program ${ }^{31}$.

For gene annotation, repeat-masked genome assembly was used to predict the protein-coding genes with the Augustus program ${ }^{32}$ trained with 250 randomly selected B. rapa genes as the reference data set. The predicted genes were validated by a blast search against the Uniprot protein database (e value threshold $<1 \mathrm{e}-05$ ). The predicted genes were validated by mapping the previously generated Illumina RNA-seq sequences ${ }^{23}$, and the RNA-seq and Iso-seq sequences generated in this study on the assembled genome. Illumina RNA-seq reads were mapped with STAR aligner ${ }^{33}$, and Iso-seq sequences were mapped using Minimap2 $\operatorname{program}^{34}$.

\subsection{Syntenic block identification and determination of gene fractionation patterns}


Syntenic regions in the assembled genome were identified with the MCScanX $\operatorname{program}^{35}$. An all-against-all Blastp comparison was carried out between the B. nigra assembly and previously reported $\mathrm{BraA}^{21}, \mathrm{BjuA}$ and $\mathrm{BjuB}^{23}$, and At genome assemblies (evalue threshold 1e-05). The blastp output file was used along with the information of positions of each gene in all the genomes for synteny analysis. Parameters for the MCScanX were set as match_score: 50, match_size: 5, gap_penalty: -1, e-value: 1e-05, max_gaps: 25 . Genes retained in each of the syntenic regions were calculated in a sliding window of 500 flanking genes at a given locus of At.

For divergence analysis, DNA sequences and the protein sequences of At genes and their orthologs in the BraA, BniB, and BjuB genomes were aligned with MUSCLE v3.8.31 software $^{36}$. Poorly aligned regions were trimmed using GBLOCKS (v0.91) ${ }^{37}$ and PAL2NAL scripts $^{38}$. A custom Perl script was used for the conversion of the aligned fasta format to the Phylip format. The Phylip files were converted into Newick format trees, and the Ks values were obtained using the PAML package ${ }^{39}$.

\section{Results}

\subsection{Genome sequencing and assembly}

The B. nigra genome was reported to be $\sim 591 \mathrm{Mb}$ in size on the basis of nuclear DNA estimations with flow cytometry ${ }^{16}$. We estimated the size of B. nigra Sangam (line BnSDH1) by using kmer frequency distribution of $\sim 40 \mathrm{x}$ Illumina PE reads. The size of the genome was estimated to be $\sim 522 \mathrm{Mb}$ (Supplementary Fig. 1). Genome sequencing of the B. nigra line BnSDH-1 on the Nanopore MinION platform yielded a total of 8,778,822 reads with an N50 value of $\sim 10 \mathrm{~kb}$ (Supplementary Table 1). The obtained long-reads provided $\sim 100 \mathrm{x}$ coverage of the $B$. nigra genome if we consider the genome size to be $\sim 522 \mathrm{Mb}$. The raw reads were assembled into 1,549 contigs with an N50 value of $\sim 1.48 \mathrm{Mb}$ using the Canu assembler (Table 1). The total size of the assembled contigs was $\sim 515.4 \mathrm{Mb}$, covering $\sim 98 \%$ of the B. nigra genome. Nanopore contigs were error-corrected with the $\sim 100 \mathrm{x}$ Illumina $\mathrm{PE}^{23}$ reads using the Pilon program for five iterative cycles. A total of 124,464 nucleotide errors and 229,767 InDels were corrected. Most of the errors, predominantly present in the noncoding regions, could be identified and corrected in the first two cycles (Supplementary Fig. 2). The quality of the error-corrected contigs was ascertained after each cycle using BUSCO 
scores. At the end of the five correction cycles, $95.4 \%$ of the gene models were found to be complete.

Optical mapping was used for finding the misassemblies in the contigs and for assembling the contigs into scaffolds. Three different libraries, one with DLE labeling and two with NLRS labeling were used for optical mapping. Mapping data obtained from each one of the three reactions were assembled by hierarchical mapping protocol to develop a consensus map (CMAP). A total of 143 contigs were found to contain misassemblies, mostly due to the merger of some of the highly conserved syntenic regions. The misassembled contigs were curated by breaking such regions at the conflicting junctions and aligning these again with the CMAP in an iterative manner. The corrected contigs could be assembled into 15 scaffolds using the CMAP (Table 1). The total size of the scaffold level assembly was $\sim 506.4 \mathrm{Mb}$, with an N50 value of $\sim 68.6 \mathrm{Mb}$. A total of 1,051 unmapped sequence fragments, encompassing $\sim 30.4 \mathrm{Mb}$ of the genome with an N50 value of $\sim 36.7 \mathrm{~kb}$, remained unscaffolded.

A genetic map of $B$. nigra, with 2,723 markers $^{23}$, was used to validate the integrity of the scaffolds and to assign these to the eight pseudochromosomes - BniB01 - BniB08 (Fig. 1). The genotyping by sequencing (GBS) based genetic markers were physically mapped on the scaffolds; no misassemblies were observed. Fourteen out of 15 scaffolds could be assembled into eight pseudochromosomes. Five out of the eight chromosomes were represented by a single scaffold each; the remaining three chromosomes consisted of two, three, and four scaffolds (Supplementary Table 2). One of the scaffolds was found to be unique as no genetic marker mapped on the scaffold; this scaffold consisted of the chloroplast genome of B. nigra. The size of the final $B$. nigra genome that could be assigned to the pseudochromosomes was $\sim 505.18 \mathrm{Mb}(\sim 96.7 \%$ of the estimated genome size).

\subsection{Genome annotation for repeat elements, centromeres, and genes}

The assembled genome was annotated for the repeat elements, centromeric repeats, and genes. A de-novo prediction approach was used for the identification of the TEs. A repeat library was developed following the steps described in the Materials and methods section. $B$. nigra genome contained $\sim 246 \mathrm{Mb}(47.12 \%)$ of repeat elements belonging to three broad categories - DNA transposons, retrotransposons, and other repeat elements. DNA transposons constituted $\sim 31 \mathrm{Mb}$ of the assembled genome; $\sim 157 \mathrm{Mb}$ of the genome was constituted of retrotransposons. LTR/Gypsy types were found to be the most predominant, $\sim 103.1 \mathrm{Mb}$ of the B. nigra genome; followed by $\sim 43.6 \mathrm{Mb}$ of LTR/Copia types 
(Supplementary Fig. 3). LTR/Copia types were found to be most abundant in the vicinity of the centromeric regions. Around $59 \mathrm{Mb}$ of the repeat elements belonged to the unknown repeat category. We earlier carried out a study of the repeat elements constituting the centromeric regions in the $\mathrm{B}$ genome of $B$. juncea ${ }^{23}$. The centromere-specific repeats were identified as highly abundant kmers in the putative centromeric regions of the BjuB genome and were characterized for their sequences and their distribution (described in details in the reference 23); identical repeats were observed to constitute the B. nigra centromeric regions (Supplementary Fig. 4).

For gene annotation, the B. nigra pseudochromosome level assembly was repeat masked and used for gene prediction with the Augustus program ${ }^{32}$ trained with $B$. rapa gene content information. A total of 57,249 protein-coding genes were predicted in the B. nigra genome. The predicted genes were validated by comparing these with the non-redundant proteins in the UniProt reference database (TrEMBL); a total of 50,233 genes could be validated at an evalue threshold of $10^{-5}$. The predicted genes were further validated by Illumina RNA seq data obtained from the seedling, leaf, and young inflorescence tissues of the line BnSDH-1 and line 2782 (Supplementary File 1). A total of 39,946 genes could be validated by the transcriptome analysis. Transcriptome sequencing was also carried out on the PacBio platform (Supplementary File 1 for all the stats and description). A total of 15,368 full-length B. nigra genes were found in the Iso-seq analysis. The Iso-seq analysis validated 2,498 additional genes. Thus, a total of 42,444 genes, out of 57,249 predicted genes were validated by the transcriptome analysis of seedling, leaf, and developing inflorescence tissues (Supplementary Fig. 5).

\subsection{Gene block arrangement in B. nigra}

The predicted 57,249 genes in B. nigra were checked for their syntenic gene block arrangements by comparisons with the gene block arrangements in the model crucifer At, and the two diploid species of the U's triangle $-B$. rapa $(\mathrm{AA})^{21}$, and $B$. oleracea $(\mathrm{CC})^{22}$ with MCScanX. The B. nigra genome was divided into 24 gene blocks (A-X), identified in $\mathrm{At}^{17}$. Three syntenic regions were identified in the B. nigra genome for each gene block in At (Supplementary Fig. 6).

Gene fractionation pattern was determined in each of the three B. nigra regions syntenic with each of the At gene blocks. Gene retention in the three syntenic regions in B. nigra was calculated by taking the number of genes present in the corresponding At gene block as a 
reference number. Based on the gene fractionation pattern, three sub-genomes were identified in the Bni genome - LF (Least Fragmented), MF1 (Moderately Fragmented), and MF2 (Most Fragmented) (Supplementary Fig. 6). In gene to gene comparison, the LF subgenome was found to contain 10,191 genes, MF1 8,822, and MF2 7,283 in comparison to a total of 19,091 genes present in the At genome. The three different syntenic regions with differential gene fractionation have been shown earlier to be a characteristic feature of the B. rapa and $B$. oleracea genomes ${ }^{13,14}$. The B. nigra genome and the B genome of B. juncea reported earlier ${ }^{23}$ show a similar pattern of gene fractionation in the three constituent paleogenomes.

The data on the physical position and the expression status of each predicted gene on the eight B. nigra pseudochromosomes Bni01 - Bni08 has been provided in the Supplementary Table 4. The data contains information on the ortholog of each At gene in the assembled $B$. nigra genome. We carried out the ortholog tagging of each gene of B. nigra and identified the nearest ortholog in B. rapa $(\mathrm{BraA})^{21}$ and B. juncea $(\mathrm{BjuB})^{23}$ genomes (Supplementary Table 4). A total of 24,799 genes were found to be BniB genome-specific; these could not be found in the syntenic regions of BraA and At genomes. Analysis of the transcriptome data showed 11,503 BniB genome-specific genes to be expressed.

\subsection{Comparison of $B$ genome pseudochromosomes of $B$. nigra and $B$. juncea}

We compared the B genome assembly of B. nigra line BnSDH-1 (BniB) with the B genome assembly of $B$. juncea line Varuna $(\mathrm{BjuB})^{23}$ for the gene content, transposable elements, centromeric repeats, and syntenic regions based on gene collinearity. The repeat content in the BniB genome ( 47.2\%) was found to be similar to that in the BjuB genome ( 51\%). The LTR/Gypsy type transposons were the most abundant TEs followed by LTR/Copia types in both the genomes. The distribution of different types of TE elements was found to be similar in both the genomes.

Earlier six B genome-specific repeats were identified in the centromeric regions of the BjuB genome $^{23}$. We found these repeats to be present in a similar manner in the centromeric regions of the B. nigra pseudochromosomes (Supplementary Fig. 4) and to be highly identical. In addition, CentBr1, CentBr2, and the other centromeric repeats reported to be present in the BraA, BolC, and BjuA genomes ${ }^{13,14,23}$ were absent in both the BjuB and BniB genomes. Our analysis indicates that the $\mathrm{B}$ genome has undergone a divergent evolutionary path than the $\mathrm{A}$ and $\mathrm{C}$ genomes in terms of the evolution of the centromeric repeats. The gene number estimation in the BniB genome $(57,249)$ is very similar to the numbers predicted in 
the BjuB genome (57,084), suggesting no significant loss of genes in the B genome after allotetraploidization. Of a total of 22,498 B genome-specific genes identified in the BjuB genome, 19,175 genes were also detected in the BniB genome.

We compared the overall genome architecture of the BniB and BjuB genomes by MCScanX based analysis. Orthologous genes were identified as the syntenic gene pairs having the least Ks value amongst all the possible combinations. The homologous gene pairs between the two B genomes were plotted using the Synmap analysis ${ }^{40}$. Very high collinearity was observed between the BniB and the BjuB pseudochromosomes (Fig. 2). An inversion was observed in each of the three pseudochromosomes - BniB01, BniB04, and BniB08 vis-à-vis the corresponding BjuB pseudochromosomes. The inversions in the BniB01 and BniB08 pseudochromsomes were found to be intra-block inversions in the $U$ and $F$ gene blocks, respectively. An inter-paleogenome non-contiguous gene block association ${ }^{23} \mathrm{~J}_{\mathrm{MF} 1}-\mathrm{I}_{\mathrm{MF1}}-\mathrm{S}_{\mathrm{MF} 2}-$ $\mathrm{S}_{\mathrm{LF}}$ observed in BjuB04 and shared with BraA04 and BolC04 was found to be $\mathrm{J}_{\mathrm{MF1}}-\mathrm{I}_{\mathrm{MF1}}$ $\mathrm{J}_{\mathrm{MF1}}-\mathrm{I}_{\mathrm{MF1}}-\mathrm{S}_{\mathrm{MF} 2}-\mathrm{S}_{\mathrm{LF}}$ in BniB04. This new gene block association in BniB04 is due to an inversion in the $\mathrm{J}_{\mathrm{MF1}}-\mathrm{I}_{\mathrm{MF1}}$. This inversion seems to be specific to the sequenced Sangam genome. It can be concluded that the progenitor B genome of B. juncea did not contain all the three inversions.

\subsection{New nomenclature for B. nigra pseudochromosomes}

Highly contiguous pseudochromosome level assemblies have been available for $B$. rapa $(\mathrm{BraA})^{21}$, and $B$. oleracea $(\mathrm{BolC})^{22}$; such an assembly is now available for $B$. nigra (BniB) allowing a chromosome level homology analysis. We carried out such an analysis for the $\mathrm{BraA}$ and $\mathrm{BniB}$ pseudochromosomes keeping the nomenclature given to the $\mathrm{BraA}^{13}$ pseudochromosomes as settled as it was the first sequenced genome from the U's triangle. Each assembled pseudochromosome of B. nigra showed homology with more than one pseudochromosome of B. rapa (Fig. 3, Supplementary Fig. 7). The size of the genomic stretches from the Bra pseudochromosomes showing homology with different Bni pseudochromosomes was calculated (Table 2). Each BniB pseudochromosome was given the number of the BraA pseudochromosome with which it shared maximum homology (except pseudochromosome BniB02). As B. nigra has eight chromosomes against ten in B. rapa, homology with BraA09 and BraA10 was not taken into consideration. The new nomenclature is Version 3. 


\section{Discussion}

The current nomenclature (Version 1) for the B. nigra LGs, recommended by the internationally agreed standard (http://www.brassica.info), is based on some early work on the comparative genetic mapping between At and B. nigra $^{41}$. A total of 160 DNA fragments from the At genome, mostly anonymous and some cDNA fragments of known genes, were used as RFLP markers. We carried out a more extensive mapping work on the A and B genomes of $B$. juncea using intron length polymorphism (IP) markers derived from the At genome $^{11}$. This allowed a more extensive comparative genetic mapping between the $\mathrm{A}$ and the B genomes of B. juncea vis-a-vis the gene block organization in the At genome. A different nomenclature (Version 2) was suggested for the BjuB genome LGs based on the extent of homology with the BjuA LGs. This nomenclature was supported by genetic mapping in $B$. juncea using RNAseq based SNP markers ${ }^{42}$.

While Version 1 and Version 2 are based on genetic mapping, Version 3 is based on gene collinearity and is, therefore, more accurate (Table 2). Version 1, due to low marker density is the most inaccurate. Version 1- B02 and B05 have no homologous regions with BraA02 and BraA05 chromosomes, respectively. Version 2 is more accurate; however, Version 2 B08 has no homology with BraA08. The inter-paleogenome non-contiguous gene block association $\mathrm{J}_{\mathrm{MF} 1}-\mathrm{I}_{\mathrm{MF1}}-\mathrm{S}_{\mathrm{MF} 2}-\mathrm{S}_{\mathrm{LF}}$, which is evidence for a common origin of the $\mathrm{A}, \mathrm{B}$, and $\mathrm{C}$ genomes $^{23}$, is only accounted for in Version 3. We propose that Version 3 nomenclature be accepted by the Brassica community both for B. nigra and B. juncea pseudochromosomes, as it reflects gene-to-gene based homology between the genomes of the species of the U's triangle.

B. nigra germplasm could be an important source for some of the major diseases afflicting the more extensively cultivated Brassica species. So far extensive efforts have been devoted to the transfer of resistance to the blackleg disease (causal organism Leptosphaeria maculans) from B. nigra to B. napus ${ }^{43}$. While the chromosomes of B. nigra containing resistance were identified in the chromosome addition lines ${ }^{44}$, actual introgression has been difficult due to limited pairing between the $\mathrm{B}$, and the $\mathrm{A}$ and $\mathrm{C}$ genome chromosomes ${ }^{45}$. Genome assemblies of the B genome in B. nigra and B. juncea have shown a very divergent chromosomal organization between the $\mathrm{B}$, and $\mathrm{A} / \mathrm{C}$ genomes. Genetic exchanges may also be limited due to a strong mechanism in the B genome for suppression of pairing between the homeologous chromosomes $^{46,47}$. 
B. nigra genome assembly reported here is an improvement over the previous $B$. nigra assemblies based on short-read sequencing ${ }^{16,48}$. The long-read Nanopore sequencing and optical mapping have provided highly contiguous genomes assembly, with five of the eight pseudochromosomes represented by a single scaffold. Centromeric and telomeric regions could also be identified.

We have compared the B. nigra (BniB) genome assembly reported in this study with the B genome of B. juncea (BjuB) assembled with SMRT sequencing and optical mapping ${ }^{23}$, and shown that the two genomes are collinear in gene arrangement, and have similar gene content and centromeric structures. We have earlier shown that the A genome of B. juncea $(\mathrm{BjuA})^{23}$ is similar to the $B$. rapa $(\mathrm{BraA})^{21}$ genome. The success of the natural allotetraploid B. juncea was therefore based on immediate stability due to suppression of homoeologous pairing between the $\mathrm{A}$ and the $\mathrm{B}$ genome as has been suggested in some of the early cytogenetic studies $^{46,47}$. However, high collinearity between BniB and BjuB genomes would allow the use of $B$. nigra germplasm for broadening the genetic base of $B$. juncea and transfer of disease resistance and other traits from B. nigra to B. juncea. As an example, B. nigra line 2782 is resistant to a number of isolates of oomycete pathogen Albugo candida and can be a useful source of resistance for the susceptible Indian gene pool lines of B. juncea ${ }^{49}$.

We have suggested a new nomenclature for the $B$. nigra LGs/chromosomes. The nomenclature currently in use does not follow any structural or evolutionary relationship with the other Brassica species of the U's triangle or At. Any nomenclature should reflect some evolutionary relationships. The new nomenclature reflects the extent of homology between the $\mathrm{B}$ genome and the $\mathrm{A}$ and $\mathrm{C}$ genomes. We propose that the suggested nomenclature for the B genome LGs/chromosomes, taking the BraA chromosome nomenclature as settled, be accepted by the Brassica researcher community.

\section{Acknowledgments}

The work was supported by the Department of Biotechnology (DBT), Government of India through two different grants - Centre of Excellence (Grant no.- BT/01/COE/08/06-II), and DBT-UDSC Partnership Centre on Genetic Manipulation of Brassicas (Grant no.BT/01/NDDB/UDSC/2016). DP acknowledges support by a J C Bose Fellowship from the Department of Science and Technology (DST) and by the Council of Scientific and Industrial Research (CSIR) as a Distinguished Scientist. 


\section{Authors contributions}

KP carried out the genome assembly, gene annotation, and the other bioinformatics analysis;

KP and DP wrote the manuscript, AKP and DP supervised the study. All the authors read and approved the final manuscript.

\section{Competing Interests}

The authors declare no competing interests.

\section{Data availability}

B. nigra genome and transcriptome sequences have been deposited under bioproject PRJNA324621 and PRJNA642332.

\section{Code availability}

Codes used in the manuscript are available on request.

\section{References}

1. Nagaharu, U. 1935, Genome analysis of Brassica with special reference to the experimental formation of B. napus and peculiar mode of fertilization. Japan J Bot, 7, 389-452.

2. Morinaga, T. 1934, Interspecific hybridization in Brassica. Cytologia, 6(1), 62-67.

3. Harberd, D.J. 1972. A contribution to the cyto-taxonomy of Brassica (Cruciferae) and its allies. Bot J Linn Soc, 65, 1-23.

4. Prakash S., Takahata Y., Kirti P.B. and Chopra VL. 1999, Cytogenetics, In: GómezCampo, C. (ed.), Biology of Brassica Coenospecies, Elsevier Science, Amsterdam, pp. 59-106.

5. Lysak, M. A. and Koch, M. A. 2011, Phylogeny, genome, and karyotype evolution of crucifers (Brassicaceae). In: Schmidt, R. and Bancroft, I. (eds), Genetics and Genomics of the Brassicaceae, Springer, New York, pp. 1-31.

6. Warwick S.I. and Black L.D. 1991, Molecular systematics of Brassica and allied genera (Subtribe Brassicinae, Brassiceae) - chloroplast genome and cytodeme congruence. Theor Appl Genet, 82, 81-92.

7. Pradhan A.K., Prakash, S., Mukhopadhyay, A. and Pental, D. 1992, Phylogeny of Brassica and allied genera based on variation in chloroplast and mitochondrial DNA patterns: molecular and taxonomic classifications are incongruous. Theor Appl Genet, $85,331-340$. 
8. Arias, T. and Pires, J. C. 2012, A fully resolved chloroplast phylogeny of the brassica crops and wild relatives (Brassicaceae: Brassiceae): Novel clades and potential taxonomic implications. Taxon, 61, 980-988.

9. Li, P., Zhang, S., Li, F., et al. 2017, A phylogenetic analysis of chloroplast genomes elucidates the relationships of the six economically important Brassica species comprising the triangle of U. Front Plant Sci, 8, 111.

10. Parkin, I. A., Gulden, S. M., Sharpe, A. G., et al. 2005, Segmental structure of the Brassica napus genome based on comparative analysis with Arabidopsis thaliana. Genetics, 171, 765-781.

11. Panjabi, P., Jagannath, A., Bisht, N., et al. 2008, Comparative mapping of Brassica juncea and Arabidopsis thaliana using Intron Polymorphism (IP) markers: homoeologous relationships, diversification and evolution of the A, B and C Brassica genomes. BMC Genomics 9, 113.

12. Lysak, M. A., Koch, M. A., Pecinka, A. and Schubert, I. 2005, Chromosome triplication found across the tribe Brassiceae. Genome Res, 15, 516-525.

13. Wang, X., Wang, H., Wang, J., et al. 2011, The genome of the mesopolyploid crop species Brassica rapa. Nat Genet, 43, 1035-1039.

14. Liu, S., Liu, Y., Yang, X., et al. 2014, The Brassica oleracea genome reveals the asymmetrical evolution of polyploid genomes. Nat Commun, 5, 3930.

15. Chalhoub, B., Denoeud, F., Liu, S., et al. 2014, Early allopolyploid evolution in the post-neolithic Brassica napus oilseed genome. Science, 345, 950-953.

16. Yang, J., Liu, D., Wang, X., et al. 2016, The genome sequence of allopolyploid Brassica juncea and analysis of differential homoeolog gene expression influencing selection. Nat Genet, 48, 1225-1232.

17. Schranz, M. E., Lysak, M. A. and Mitchell-Olds, T. 2006, The ABC's of comparative genomics in the Brassicaceae: building blocks of crucifer genomes. Trends Plant Sci, $11,535-542$.

18. Lysak, M. A., Mandakova, T. and Schranz, M. E. 2016, Comparative paleogenomics of crucifers: ancestral genomic blocks revisited. Curr Opin Plant Biol, 30, 108-115.

19. Tang, H., Woodhouse, M. R., Cheng, F., et al. 2012, Altered patterns of fractionation and exon deletions in Brassica rapa support a two-step model of paleohexaploidy. Genetics 190, 1563-74.

20. Sharma, S., Padmaja, K. L., Gupta, V., Paritosh, K., Pradhan, A. K. and Pental, D. 2014, Two plastid DNA lineages - Rapa/Oleracea and Nigra - within the tribe Brassiceae can be best explained by reciprocal crosses at hexaploidy: evidence from divergence times of the plastid genomes and R-block genes of the A and B genomes of Brassica juncea. PLoS One, 9, e93260.

21. Zhang, L., Cai, X., Wu, J., et al. 2018, Improved Brassica rapa reference genome by single-molecule sequencing and chromosome conformation capture technologies. Hort Res, 5, 1-11.

22. Belser, C., Istace, B., Denis, E., et al. 2018, Chromosome-scale assemblies of plant genomes using nanopore long reads and optical maps. Nat Plants, 4, 879-887. 
23. Paritosh, K., Yadava, S.K., Singh, P. et al. 2019, A chromosome-scale assembly of allotetraploid Brassica juncea (AABB) elucidates comparative architecture of the A and B genomes. bioRxiv, p.681080.s

24. Rogers, S.O. and Bendich, A.J. 1994, Extraction of total cellular DNA from plants, algae and fungi. In: Gelvin, S.B. and Schilperoort, R.A. (eds.), Plant Molecular Biology Manual, Springer, Dordrecht, pp-183-190.

25. Marçais, G. and C. Kingsford. 2011, A fast, lock-free approach for efficient parallel counting of occurrences of k-mers. Bioinformatics 27: 764-770.

26. Sun, H., Ding, J., Piednoël, M. and Schneeberger K. 2018, findGSE: estimating genome size variation within human and Arabidopsis using k-mer frequencies. Bioinformatics 34: 550-557.

27. Koren, S., Walenz, B.P., Berlin, K., Miller, J.R., Bergman, N.H. and Phillippy, A.M. 2017, Canu: scalable and accurate long-read assembly via adaptive k-mer weighting and repeat separation. Genome Res 27: 722-736.

28. Li, H. and Durbin R. 2009, Fast and accurate short read alignment with BurrowsWheeler transform. Bioinformatics 25: 1754-1760.

29. Walker, B.J., Abeel, T., Shea, T., et al. 2014, Pilon: an integrated tool for comprehensive microbial variant detection and genome assembly improvement. PLoS One, 9, e112963.

30. Simão, F.A, Waterhouse, R.M, Ioannidis, P, Kriventseva, E.V. and Zdobnov, E.M. 2015, BUSCO: assessing genome assembly and annotation completeness with singlecopy orthologs. Bioinformatics, 31, 3210-3212.

31. Xu, Z. and Wang, H. 2007, LTR_FINDER: an efficient tool for the prediction of fulllength LTR retrotransposons. Nucleic Acids Res, 35, W265-268.

32. Stanke, M. and Morgenstern, B. 2005, AUGUSTUS: a web server for gene prediction in eukaryotes that allows user-defined constraints. Nucleic Acids Res 33: W465-467.

33. Dobin, A., Davis, C.A., Schlesinger, F., et al. 2013, STAR: ultrafast universal RNAseq aligner. Bioinformatics 29: 15-21.

34. Li, H. 2018, Minimap2: pairwise alignment for nucleotide sequences. Bioinformatics 34: 3094-3100.

35. Wang, Y., Tang, H., Debarry, J. D., et al. 2012, MCScanX: a toolkit for detection and evolutionary analysis of gene synteny and collinearity. Nucleic Acids Res, 40, e49.

36. Edgar, R.C. 2004, MUSCLE: multiple sequence alignment with high accuracy and high throughput. Nucleic Acids Res 32, 1792-7.

37. Talavera, G. and Castresana, J. 2007, Improvement of phylogenies after removing divergent and ambiguously aligned blocks from protein sequence alignments. Syst Biol 56, 564-77.

38. Suyama, M., Torrents, D. and Bork, P. 2006, PAL2NAL: robust conversion of protein sequence alignments into the corresponding codon alignments. Nucleic Acids Res 34, W609-12.

39. Yang, Z. 2007, PAML 4: phylogenetic analysis by maximum likelihood. Mol Biol Evol, 24, 1586-1591. 
40. Haug-Baltzell, A., Stephens, S.A., Davey, S., Scheidegger, C.E. and Lyons, E. 2017, SynMap2 and SynMap3D: web-based whole-genome synteny browsers. Bioinformatics, 33, 2197-2198.

41. Lagercrantz, U. 1998, Comparative mapping between Arabidopsis thaliana and Brassica nigra indicates that Brassica genomes have evolved through extensive genome replication accompanied by chromosome fusions and frequent rearrangements. Genetics, 150, 1217-1228.

42. Paritosh, K., Gupta, V., Yadava, S. K., Singh, P., Pradhan, A. K. and Pental, D. 2014, RNA-seq based SNPs for mapping in Brassica juncea (AABB): synteny analysis between the two constituent genomes A (from B. rapa) and B (from B. nigra) shows highly divergent gene block arrangement and unique block fragmentation patterns. BMC Genomics, 15, 396.

43. Delourme, R., Barbetti, M., Snowdon, R., et al. 2011, Genetics and genomics of disease resistance. In: Edwards, T., Batley, J., Parkin, I. and Kole C. (eds.), Genetics, Genomics and Breeding of Oilseed Brassicas. CRC Press, Boca Raton, pp.276-318.

44. Chevre, A.M., Eber, F., This, P., et al. 1996, Characterization of Brassica nigra chromosomes and of blackleg resistance in B. napus - B. nigra addition lines. Plant Breeding, 115, 113-118.

45. Gaebelein, R., Alnajar, D., Koopmann, B. and Mason, A.S. 2019, Hybrids between Brassica napus and B. nigra show frequent pairing between the $\mathrm{B}$ and $\mathrm{A} / \mathrm{C}$ genomes and resistance to blackleg. Chromosome Res., 27, 221-236.

46. Attia, T. and Röbbelen, G. 1986. Cytogenetic relationship within cultivated Brassica analyzed in amphihaploids from the three diploid ancestors. Can J Genet Cytol, 28, 323-329.

47. Busso, C., Attia, T. and Röbbelen, G. 1987. Trigenomic combinations for the analysis of meiotic control in the cultivated Brassica species. Genome, 29, 331-333.

48. Wang, W., Guan, R., Liu, X., et al. 2019, Chromosome level comparative analysis of Brassica genomes. Plant Mol Biol, 99, 237-249.

49. Arora, H., Padmaja, K.L., Paritosh, K., Mukhi, N. et al. 2019. BjuWRR1, a CC-NBLRR gene identified in Brassica juncea, confers resistance to white rust caused by Albugo candida. Theor Appl Genet, 132, 2223-2236. 


\section{Figure Legends}

Fig. 1. Graphic representation of the Brassica nigra pseudochromosomes. Each chromosome is represented by a vertical bar. Each horizontal bar represents a gene. Gene blocks have been identified on the basis of synteny with the A. thaliana gene blocks (A-X), as defined and color-coded by Schranz et $\mathrm{al}^{17}$. Centromeric repeats are represented as black dots and telomeric repeats as red dots. A new nomenclature has been given to the $B$. nigra pseudochromosomes on the basis of maximum gene-level collinearity with the B. rapa pseudochromosomes $^{21}$.

Fig. 2. Comparison of B. nigra (BniB) pseudochromosomes with B. juncea $B$ genome (BjuB) pseudochromosomes. The comparison was carried out with the Synfind program available at the CoGe website. Gene pairs with the least Ks value were identified as orthologous genes between the two genomes. Strictly orthologous genes have been denoted as blue dots, other syntenic regions are shown with the green dots. Very high gene collinearity was observed between the two B genomes, except for the three inversions in the B. nigra pseudochromosomes - BniB01, BniB04, and BniB08. Centromeric regions are devoid of genes and therefore, recognized as gaps. The nomenclature of the Bni pseudochromosomes is according to the new nomenclature, the BjuB pseudochromosome nomenclature is following Panjabi et $\mathrm{al}^{11}$.

Fig. 3. Comparative gene block arrangements in $B$. rapa ${ }^{21}$, B. nigra (this study), and $B$. oleracea $^{22}$. All the three assemblies are with long-read sequences. The LF, MF1 and MF2 paleogenomes present in the $\mathrm{A}, \mathrm{B}$ and $\mathrm{C}$ genomes have been represented by red, green and blue colors, respectively. The $\mathrm{A}$ and $\mathrm{C}$ genomes show more similarity in gene block arrangements, whereas the B genome has divergent arrangements. 
Table 1. Genome assembly statistics of $B$. nigra variety Sangam

\begin{tabular}{|c|c|c|c|}
\hline Oxford Nanopore & 回 & 国 & 国 \\
\hline BioNano & & 圆 & 圆 \\
\hline \multirow[t]{2}{*}{ Linkage Map } & & & 圆 \\
\hline & \multicolumn{3}{|c|}{ B. nigra $(\mathrm{BB}, 2 \mathrm{2n}=8)$} \\
\hline Total assembly size & $515,400,203$ & - & - \\
\hline Number of contigs & 1,549 & - & - \\
\hline Longest contig & $17,509,570$ & - & - \\
\hline N50 contig length & $1,488,221$ & - & - \\
\hline Number of scaffolds & - & 15 & - \\
\hline Total scaffold size & - & $506,396,041$ & - \\
\hline Longest Scaffolds & - & $115,616,497$ & - \\
\hline N50 scaffolds length & - & $68,578,869$ & - \\
\hline Unscaffolded contigs & - & 1,051 (partial) & - \\
\hline Number of pseudochromosomes/LGs & - & - & 8 \\
\hline Scaffolds assigned to LGs & - & - & 14 \\
\hline Contigs assigned to LGs & - & - & - \\
\hline Unassigned scaffolds to LGs & - & - & - \\
\hline Unassigned contigs to LGs & - & - & - \\
\hline Length of assigned sequences to LGs & - & - & $505,183,631$ \\
\hline Length of unassigned sequences to LGs & - & - & $30,296,383$ \\
\hline N50 length pseudochromosomes (bp) & - & - & $63,988,665$ \\
\hline
\end{tabular}


Table 2. The size of the genomic stretches from the B. rapa pseudochromosomes showing gene collinearity-based homology with different $B$. nigra pseudochromosomes

\begin{tabular}{|c|c|c|c|c|c|c|c|c|c|}
\hline \multirow{14}{*}{ 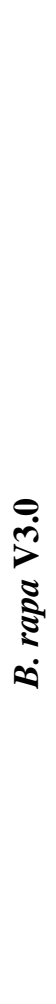 } & & \multicolumn{8}{|c|}{ B. nigra } \\
\hline & BniB - V1 & B01 & B06 & B02 & B07 & B04 & B05 & B08 & B03 \\
\hline & BjuB - V2 & В02 & B06 & B03 & B05 & B04 & B08 & B07 & B01 \\
\hline & BniB - V3 & B01 & B02 & B03 & B04 & B05 & B06 & B07 & B08 \\
\hline & A01 & $\begin{array}{l}15185553 \\
\mathbf{5 1 . 3 \%}\end{array}$ & & & & & & & $\begin{array}{l}13228780 \\
\mathbf{4 4 . 7 \%}\end{array}$ \\
\hline & A02 & $\begin{array}{l}16418817 \\
\mathbf{5 2 . 2 \%}\end{array}$ & $\begin{array}{l}14535932 \\
\mathbf{4 6 . 2 \%}\end{array}$ & & & & & & \\
\hline & A03 & & & $\begin{array}{l}15196210 \\
\mathbf{3 9 . 9 \%}\end{array}$ & & & $\begin{array}{l}14502023 \\
\mathbf{3 8 \%}\end{array}$ & $\begin{array}{l}1218940 \\
\mathbf{3 . 2 \%}\end{array}$ & $\begin{array}{l}4307164 \\
\mathbf{1 1 . 3 \%}\end{array}$ \\
\hline & A04 & & & & $\begin{array}{l}14898239 \\
67.9 \%\end{array}$ & $\begin{array}{l}5900409 \\
\mathbf{2 6 . 9 \%}\end{array}$ & & $\begin{array}{l}702255 \\
\mathbf{3 . 2 \%}\end{array}$ & \\
\hline & A05 & & & & $\begin{array}{l}10591962 \\
\mathbf{3 7 . 2 \%}\end{array}$ & $\begin{array}{l}13872273 \\
\mathbf{4 8 . 7 \%}\end{array}$ & & $\begin{array}{l}3293639 \\
\mathbf{1 1 . 5 \%}\end{array}$ & \\
\hline & A06 & & $\begin{array}{l}7524595 \\
19.1 \% \\
\end{array}$ & $\begin{array}{l}5158622 \\
\mathbf{1 7 . 6 \%} \\
\end{array}$ & & $\begin{array}{l}7315564 \\
\mathbf{2 4 . 3 \%}\end{array}$ & $\begin{array}{l}8704851 \\
\mathbf{2 9 . 8 \%}\end{array}$ & & \\
\hline & A07 & & & & $\begin{array}{l}2818377 \\
\mathbf{9 . 7 \%}\end{array}$ & $\begin{array}{l}7270483 \\
\mathbf{2 5 . 2 3 \%}\end{array}$ & & $\begin{array}{l}15688850 \\
\mathbf{5 4 . 5 \%}\end{array}$ & \\
\hline & A08 & & & & & & & $\begin{array}{l}11410503 \\
\mathbf{4 9 . 7 \%}\end{array}$ & $\begin{array}{l}11463772 \\
\mathbf{4 9 . 9 \%}\end{array}$ \\
\hline & A09 & & $\begin{array}{l}21384087 \\
\mathbf{4 7 . 3 \%}\end{array}$ & $\begin{array}{l}10792098 \\
\mathbf{2 3 . 9 \%}\end{array}$ & & $\begin{array}{l}126317 \\
\mathbf{0 . 2 7 \%}\end{array}$ & $\begin{array}{l}2342318 \\
\mathbf{5 . 1 8 \%}\end{array}$ & $\begin{array}{l}1203054 \\
2.7 \%\end{array}$ & $\begin{array}{l}3813925 \\
8.4 \%\end{array}$ \\
\hline & A10 & & $\begin{array}{l}2004504 \\
\mathbf{9 . 7 \%}\end{array}$ & & & $\begin{array}{l}2508933 \\
\mathbf{1 2 . 1 \%}\end{array}$ & $\begin{array}{l}14954862 \\
\mathbf{7 2 . 1 \%}\end{array}$ & $\begin{array}{l}887503 \\
\mathbf{4 . 2 \%}\end{array}$ & \\
\hline
\end{tabular}

* V1- BniB LG nomenclature by Lagercrantz et al, ${ }^{41}$ based on genetic mapping; V2- BjuB LG nomenclature by Panjabi et al, ${ }^{11}$ based on genetic mapping; V3- BniB pseudochromosome nomenclature proposed in this study based on the long-read genome assembly

**Explanation of the numbers in the peach colored boxes- As an example - pseudochromosome A01 of $B$. rapa has homology with two B genome chromosomes; a region of $15,185,553 \mathrm{bp}(51.3 \%$ of the total length of A01) with one of the B genome pseudochromosome and a region of $13,228,780 \mathrm{bp}(44.7 \%$ of the total length of A01) with the other. 







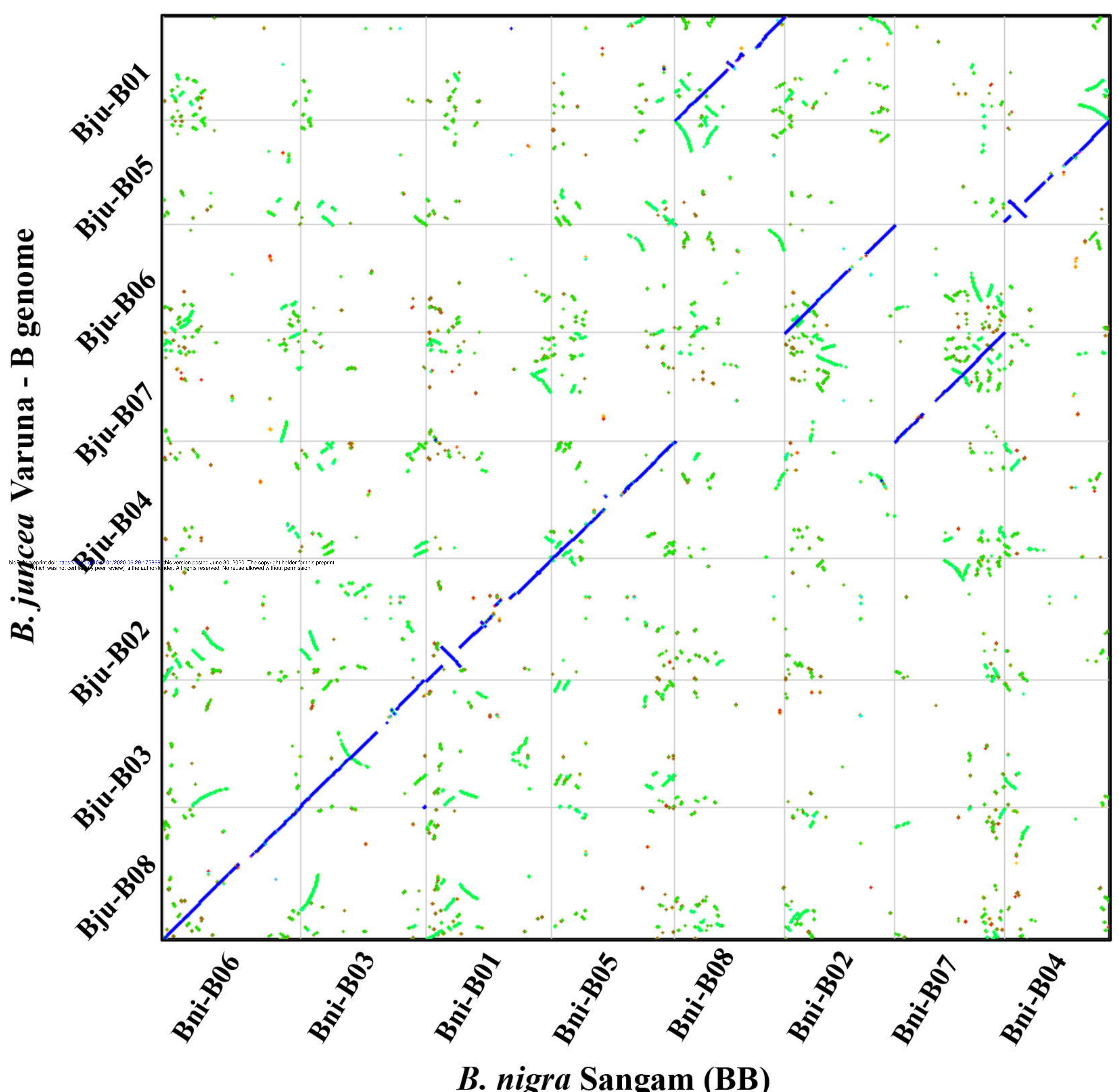


B. oleracea (CC)

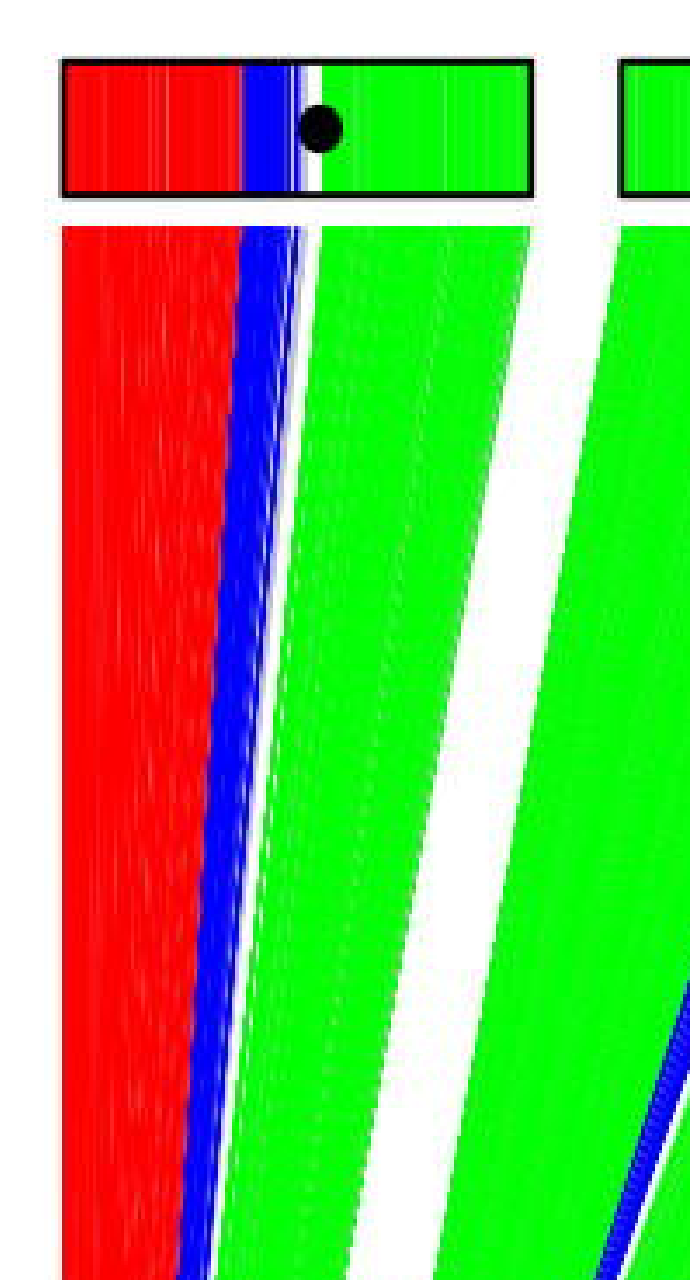

B. rapa (AA)

B. nigra (BB)

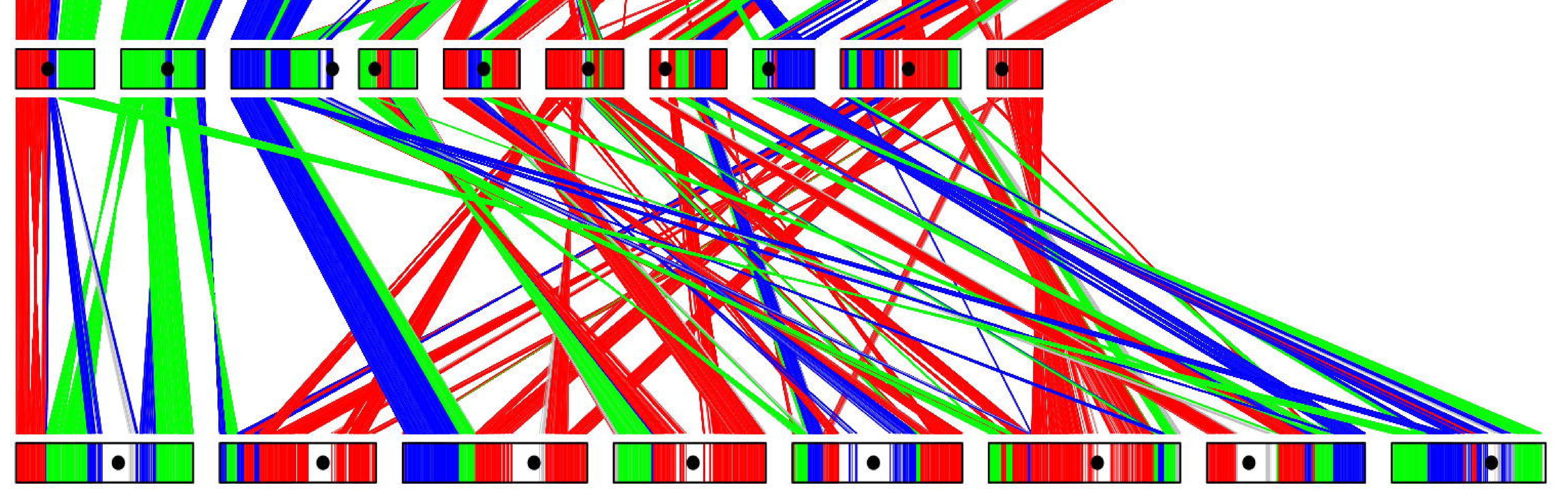

